\title{
SISTEMA DE MODULAÇÃO CODIFICADA MULTINÍVEL COM CÓDIGOS DE BLOCO BINÁRIOS
}

\author{
Emílio C.G.Wille e Walter Godoy Júnior \\ CPGEI - CEFET-PR \\ Av. Sete de Setembro, 3165 - Curitiba, PR - CEP 80230/901 \\ E-mail: godoy@cpgei.cefetpr.br
}

\begin{abstract}
Resumo: Neste trabalho é proposto um sistema original de obtenção de códigos apropriados para a modulação codificada multinível. Estes códigos são formados a partir de códigos de bloco binários curtos e são encontrados via busca computacional. Alguns desses códigos são apresentados. A decodificação dos códigos propostos é facilitada devido a sua representação binária. É realizada uma comparação com os códigos de Sayegh e com os de Baldini. São apresentados resultados de simulação da decodificação de alguns códigos obtidos.
\end{abstract}

Abstract: This paper proposes an original system to the attainment of multilevel coded modulation proper codes. These codes are made up from short binary block codes and are found through computer search. Some of these zodes are shown here. The breaking of the proposed codes is easy, since they are in the binary representation. A comparation with the Sayegh and Baldini codes is made and code breaking simulation results of some codes are shown.

Palayras Chave: modulação codificada, códigos sorretores de erro.

\section{INTRODUÇÃO}

A modulação codificada é uma técnica que permite a :edução da taxa de erro de bit em sistemas de transmissão ¿̇e dados que operam em canais ruidosos limitados em ¿̇ixa de freqüência, sem alterar a taxa de transmissão de רformação [1]. Sistemas que utilizam códigos de bloco zorretores de erro são conhecidos por sistemas de Modulação Codificada com Códigos de Bloco (BCM). Os ¿̇senvolvimentos nesta área se iniciaram com Imai e Trakawa [2], Cusack [3] e foram generalizados por Sayegh [4]. Recentemente, Baldini [5] apresentou uma sova abordagem do problema.

Sayegh mostrou como determinar códigos de bloco binários necessários à construção de códigos ótimos no ¿jpaço de sinais. Estes códigos são obtidos pelo mapeamento de bits de palavras-código de códigos de

Publicação recomendada pelo Editor de Área de Teoria das Comunicaçōes. bloco binários para os sinais de modulação. No trabalho de Baldini são propostos códigos de bloco multiníveis para a modulação Q-PSK codificada obtidos por busca computacional.

Este trabalho apresenta um novo sistema para a obtenção de códigos para modulação codificada. Estes códigos são obtidos pelo mapeamento de conjunto de bits, pertencentes a palavras-código de códigos binários transformadas linearmente, em sinais de modulação.

É apresentada, também, a representação do sistema por um único código de bloco binário, cujo mapeamento para o espaço de sinais, permite a maximização da distância Euclidiana mínima entre os sinais codificados. A busca destes códigos é feita computacionalmente, e algumas restrições são impostas de modo a diminuir o tempo gasto neste processo.

\section{CÓDIGOS DE SAYEGH}

Seja S o conjunto de $Q$ sinais da modulação bidimensional adotada:

A cada sinal é associada uma seqüência de bits distinta:

$$
\begin{aligned}
& S=\left\{s_{1}(t), s_{2}(t), \ldots, s_{Q}(t)\right\} \\
& B_{i}=\left(b_{1 . j}, b_{2 . i}, \ldots, b_{m+1 . i}\right), \quad i=1, \ldots, Q
\end{aligned}
$$

onde $Q=2^{\mathrm{m}+1}$.

Esta associação segue a regra do mapeamento por partição de conjunto de Ungerboeck [1]. A codificação é descrita a seguir. Seja A um arranjo-código $(\mathbf{m}+1) \times \mathbf{n}$ binário, onde $\mathbf{n}$ é o comprimento das palavras-código:

$$
\mathbf{A}=\left|\begin{array}{cccc}
\mathrm{a}_{1.1} & \mathrm{a}_{1,2} & \cdots & \mathrm{a}_{1, \mathrm{n}} \\
\mathrm{a}_{2,1} & \mathrm{a}_{2.2} & \cdots & \mathrm{a}_{2, \mathrm{n}} \\
\vdots & \vdots & & \vdots \\
a_{\mathrm{m}+1,1} & \mathrm{a}_{\mathrm{m}+1,2} & \cdots & \mathrm{a}_{\mathrm{m}+1 . \mathrm{n}}
\end{array}\right|
$$

Cada linha de $\mathbf{A}$ é constituída por uma palavra-código de um código de bloco binário $\mathbf{C}_{i}\left(\mathbf{n}, \mathbf{k}_{i}, \mathbf{d}_{\mathrm{i}}\right)$, onde $\mathbf{k}_{\mathrm{i}}$ é o número de bits de informação e $\mathrm{d}_{\mathrm{i}}$ a distância mínima de 
Hamming do código. Cada coluna de A é associada a um sinal $s_{j}(t)$ do conjunto $S$. O conjunto de sinais transmitidos é dado por:

$$
\mathrm{s}_{\mathrm{j}}(\mathrm{t})=\left\{\mathrm{s}_{\mathrm{j} 1}(\mathrm{t}), \mathrm{s}_{\mathrm{j} 2}(\mathrm{t}), \ldots, \mathrm{s}_{\mathrm{jn}}(\mathrm{t})\right\}
$$

A distância Euclidiana mínima ao quadrado entre os zonjuntos de sinais codificados é dada por:

$\mathrm{d}_{\mathrm{E}}{ }^{2}=\min \left(\mathrm{d}_{\mathrm{l}} \cdot \Delta_{1}{ }^{2}, \mathrm{~d}_{2}, \Delta_{2}^{2}, \ldots, \mathrm{d}_{\mathrm{m}+1} \cdot \Delta_{\mathrm{m}+1}{ }^{2}\right)$

onde $\Delta_{\mathrm{i}}$ é a distância Euclidiana mínima em cada nível da partição de conjuntos da constelação usada

O ganho assintótico de codificação é calculado em relação a um sistema de referência e é dado por:

$$
\mathrm{g}_{\infty}=10 \log _{10}\left(\mathrm{~d}_{\mathrm{E}}{ }^{2} / \mathrm{d}_{\mathrm{Er}_{\mathrm{r}}}{ }^{2}\right)
$$

כistema de referência não-codificado possui $Q / 2$ sinais le modo a manter a mesma taxa de modulação do sistema zodificado, ou seja, $\mathbf{m}$ bits por símbolo. A decodificação to arranjo-código, proposta por Sayegh, é realizada linhaa-linha com o uso de $(\mathbf{m}+1)$ decodificadores, o que torna o processo sub-ótimo.

\section{CÓDIGOS DE BALDINI}

O sistema proposto por Baldini [5] representa uma alteraçāo quanto à filosofia original da modulação codificada, realizando primeiro o mapeamento da sequuência binária de informação no conjunto expandido de sinais de modulação e, em seguida, codificando-os para introduzir redundância.

A Figura 3.1 mostra a estrutura do codificador de Baldini. A saída paralela da fonte binária é composta por $(\mathbf{m}+1)$ bits de informação que são mapeados em um $\operatorname{dos} Q=$ $2^{m+1}$ elementos $u_{i}$ pertencentes ao conjunto $Z_{Q}$ de sinais da modulação $\mathbf{Q}-\mathrm{PSK}$. $\mathbf{Z}_{\mathrm{Q}}$ é um anel de inteiros módulo- $\mathbf{Q}$, $\mathrm{Z}_{\mathrm{Q}}=\{0,1, \ldots, \mathrm{Q}-1\}$. A entrada do codificador multinível é a seqüência $\mathbf{U}=\left(\mathrm{u}_{1}, \mathrm{u}_{2}, \ldots, \mathrm{u}_{\mathrm{k}}\right)$ de $\mathbf{k}$ elementos de $\mathbf{Z}_{\mathrm{Q}}$. $A$ jequiência $\mathbf{U}$ é codificada pelo codificador multinível transformando-se na seqüência $\mathbf{V}=\left(\mathrm{v}_{1}, \mathrm{v}_{2}, \ldots, \mathrm{v}_{\mathrm{n}}\right)$, com $\mathbf{n}$ $\geq \mathbf{k}$, também de elementos de $Z_{\mathrm{Q}}$.

O problema é determinar a estrutura do código multinível Je modo a maximizar a distância Euclidiana mínima entre is palavras-código. Baldini determinou uma classe de zódigos lineares chamada códigos pseudo-cíclicos nultiníveis devido à sua estrutura matricial e propriedades semelhantes às dos códigos binários cíclicos. Estes são obtidos por busca computacional, e permitem ganhos assintóticos de codificaçăo de 3 a $6 \mathrm{~dB}$ para modulação PSK codificada em relação à modulaçāo PSK não codificada.

Embora apresentem propriedades interessantes, como por exemplo a superlinearidade [5], estas, aparentemente, não são suficientes para a determinação de um processo simples de decodificação por decisão suave.

\section{SISTEMA PARA OBTENÇÃO DE CÓDIGOS MULTINIVEIS}

A Figura 4.1 mostra o sistema proposto neste trabalho.

A entrada do codificador é a sequência de informação $\mathbf{U}=$ $\left(u_{1}, u_{2}, \ldots, u_{k}\right)$ de elementos $u_{j}$ pertencentes ao conjunto $\mathrm{D}_{\mathrm{Q}}$ de sinais de modulação $\mathrm{Q}$-PSK. $\mathrm{D}_{\mathrm{Q}}$ é um conjunto de inteiros de $\mathbf{Q}$ elementos, $\mathbf{D}_{\mathbf{Q}}=(0,1 \ldots . \mathbf{Q}-1)$, onde $\mathbf{Q}=$ $2^{m+1}$. O conversor multinível/binário converte a seqüência de informação $\mathbf{U}$ em um conjunto de $(\mathbf{m}+1)$ vetores binários $\mathrm{E}_{\mathrm{i}}=\left(\mathrm{e}_{\mathrm{i} 1}, \mathrm{e}_{\mathrm{i} 2}, \ldots, \mathrm{e}_{\mathrm{ik}}\right)$. A relação adotada entre os elementos $u_{j}$ e os dígitos binários $e_{1}$ é a seguinte (mas qualquer mapeamento bi-unívoco serve a esta finalidade):

$$
\begin{aligned}
& u_{j} 0123 \\
& \mathrm{e}_{1 \mathrm{j}} 010101 \\
& e_{2 j} 0011 \\
& \mathbf{m}=2 \quad \begin{array}{lllllll}
u_{j} & 0 & 1 & 234 & 56
\end{array} \\
& e_{2 j} 00101110 \\
& \mathrm{e}_{3 \mathrm{j}} 00010111
\end{aligned}
$$

Os vetores $\mathbf{E}_{\boldsymbol{i}}$ são, então, codificados $\mathrm{cm}$ palavra-código do código binário $\mathbf{C}(\mathbf{n}, \mathbf{k} . \mathbf{d})$ de matriz hınária geradora $\mathbf{G}$, $\mathbf{k} \times \mathbf{n}$, (eq. 4.1), pelos codificadores CD. Os sejetores SL escolhem $\mathbf{k}$ bits que são somados (soma midulo dois) aos demais bits selecionados e multiplicam as matrizes de transformação biárias MT, $\mathbf{k} \times \mathbf{n},(\mathrm{eq} q$ \$.2) A saida do iésimo somador módulo-2, $\mathrm{i} \geq 2$, alımenta a entrada do bloco MT correspondente e alimenis tamtion uma das duas entradas do $(i+1)$-ésimo somadur midulo-2. cuja outra entrada é provida pela saída do $(1+11$-isımo seletor SL.

$$
\begin{aligned}
& \mathbf{G}=\left|\begin{array}{l:l}
\mathbf{I} & \mathbf{P}
\end{array}\right| \\
& \mathbf{M T}=\left|\begin{array}{l:l}
\mathbf{0} & \mathbf{M}
\end{array}\right|
\end{aligned}
$$

Os bits resultantes após os blocos MT são somados às

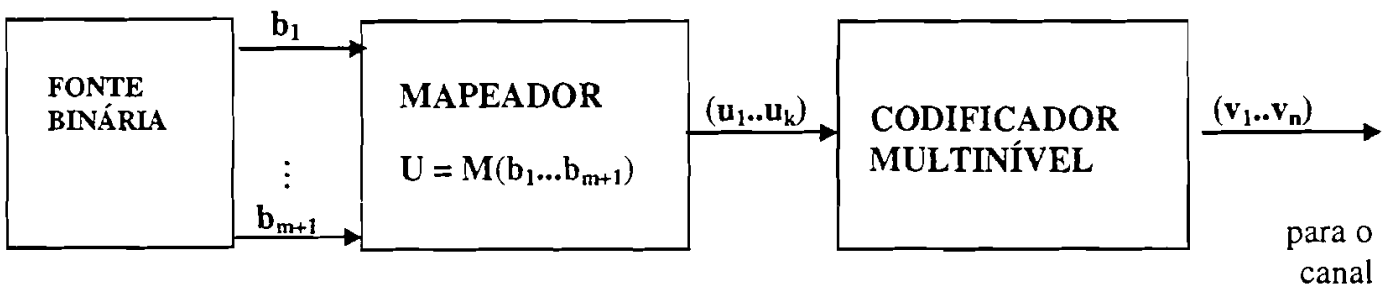

Figura 3.1 - Estrutura do Codificador de Baldini. 


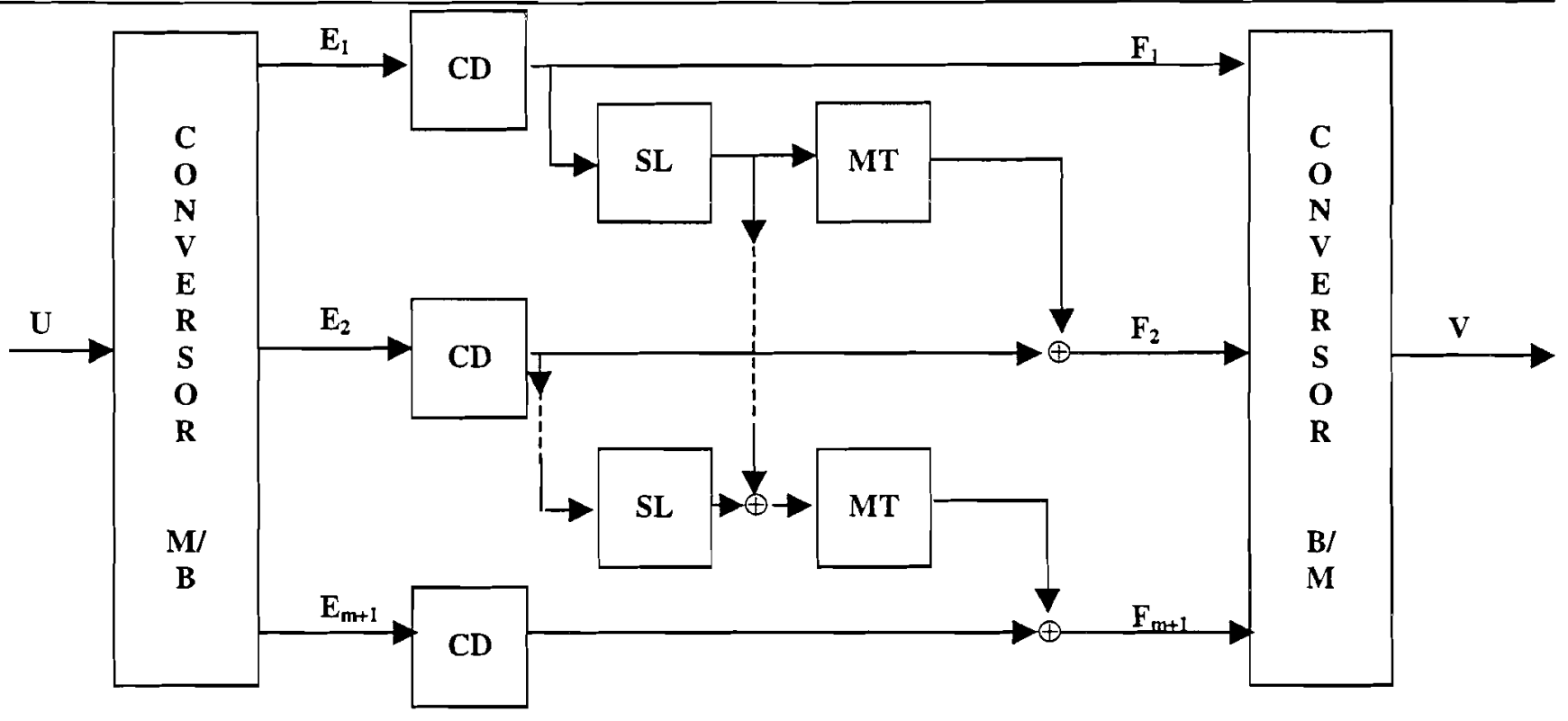

Figura 4.1 - O Sistema Proposto.

Fảavras-código provenientes da codificação dos vetores $E_{\text {: }}$. Ao final destas operações, os elementos dos vetores $F_{i}$ $=\left(f_{i 1}, f_{i 2}, \ldots, f_{i n}\right)$ resultantes, são convertidos em elementos do conjunto de inteiros sendo mapeados para os sinais da modulação. Assim, a seqüência $\mathbf{V}$ pode ser vista como uma palavra-código multinível.

As matrizes MT são encontradas por busca computacional, mantendo fixa a matriz $\mathbf{G}$, de modo que a distância Euclidiana mínima do código multinível obtido seja a maior possível. Os códigos lineares multiníveis obtidos possuem taxa $\mathbf{R}=\mathbf{k} / \mathbf{n}$, e o limite inferior para a distância Euclidiana mínima ao quadrado é dado por [6.7]:

$$
\mathrm{d}_{\mathrm{E}}^{2} \geq \mathrm{d}_{1} \cdot \Delta_{1}^{2}
$$

onde $\Delta_{1}$ é a distância Euclidiana mínima entre pontos na constelação e $\mathbf{d}$ é a distância de Hamming mínima do código de matriz geradora $\mathbf{G}$.

\section{REPRESENTAÇÃO DO SISTEMA POR UMA ÚNICA MATRIZ}

Pode-se representar todo 0 funcionamento da parte binária do sistema da Figura 4.1 através de uma única matriz binária. Essa representação só é válida quando os códigos $\mathbf{C}(\mathbf{n}, \mathbf{k}, \mathbf{d})$ usados possuem mesmo comprimento $\mathbf{n}$ e mesmo número de símbolos de informação $\mathbf{k}$.

Considere-se que a modulação é a 4-PSK, que SL seleciona os $\mathbf{k}$ primeiros bits da palavra binária codificada e sejam as matrizes G e MT mostradas anteriormente, então o funcionamento da parte binária do sistema é zepresentado pela matriz $\mathbf{G}^{\prime}, 2 \mathbf{k} \times 2 \mathrm{2n}$, dada por :

$$
\mathbf{G}^{\prime}=\left|\begin{array}{l:l:l}
\mathbf{I} & \mathbf{P} & \mathbf{M} \\
\hdashline & \mathbf{0} & \mathbf{P} \\
\end{array}\right|
$$

onde a submatriz I é a identidade, $\mathrm{P}$ e $\mathbf{M}$ são provenientes das matrizes $\mathbf{G}$ e $\mathbf{M T}$, e $\mathbf{0}$ é a submatriz nula. Nota-se que a coluna formada pelas submatrizes $\mathbf{P}$ e $\mathbf{0}$ representa as operaçōes ocorridas na primeira linha do sistema da figura 4.1 , e a segunda coluna representa operações na segunda linha. Esta idéia pode ser generalizada para modulações de maior cardinalidade.

Para a codificação, utilizando a matriz $G^{\prime}$, deve-se obter um vetor informação que corresponde à seqüência de vetores binários $\mathbf{E}_{\mathrm{i}}$, assim :

$$
U^{\prime}=\left[E_{1} E_{2} \ldots E_{m+1}\right.
$$

Após a multiplicação pela matriz G' obtém-se uma palavra-código binária $V^{\prime}$ dada abaixo:

$$
V^{\prime}=\left[\begin{array}{lllllll}
E_{1} & E_{2} & \ldots & E_{m+1} & P_{1} & P_{2} & \ldots \\
P_{m+1}
\end{array}\right]
$$

onde $\mathbf{P}_{\mathrm{i}}=\left(\mathrm{p}_{\mathrm{i} 1}, \mathrm{p}_{\mathrm{i} 2}, \ldots, \mathrm{p}_{\mathrm{i}(\mathrm{n}-\mathrm{k})}\right)$ são vetores de bits de paridade.

O arranjo-código correspondente ao sistema é :

$$
\mathbf{A}^{\prime}=\left|\begin{array}{c:c}
\mathbf{E}_{1} & \mathbf{P}_{1} \\
\mathbf{E}_{2} & \mathbf{P}_{2} \\
\vdots & \vdots \\
\mathbf{E}_{m+1} & \mathbf{P}_{m+1}
\end{array}\right|
$$

Observa-se que o arranjo-código $\mathbf{A}^{\prime}$ é obtido de uma única palavra-código binária pertencente a um código binário $\mathbf{C}\left[(\mathbf{m}+1) \mathbf{n},(\mathbf{m}+1) \mathbf{k}, \mathbf{d}^{\prime}\right]$, com taxa de codificação $\mathbf{R}$ $=\mathbf{k} / \mathbf{n}$. 
Cada coluna do arranjo-código é convertida para um elemento do conjunto de inteiros de $\mathbf{Q}$ elementos. Cada elemento é transmitido considerando-se uma modulação Q-PSK.

Assim, considerando-se que o arranjo-código transporta toda a informação necessária à decodificação, é lógico pensar-se em decodificá-lo todo de uma só vez (usando um único decodificador) com aproveitamento máximo de informação, e não linha-a-linha de forma sub-ótima.

\section{ESTRUTURA DA MATRIZ GERADORA}

Resta determinar a matriz geradora, deste novo código binário $\mathbf{C}\left[(\mathbf{m}+1) \mathbf{n},(\mathbf{m}+\mathbf{l}) \mathbf{k}, \mathbf{d}^{\prime}\right]$ de modo a maximizar a distância Euclidiana mínima entre todos os sinais codificados. A estrutura utilizada para as modulações 4PSK e 8-PSK é a seguinte :

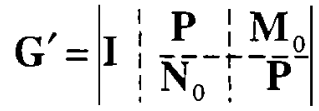

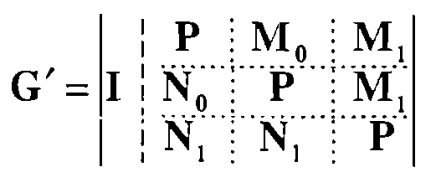

Estas matrizes são compostas por sub-matrizes cíclicas $\mathbf{k} \times(\mathbf{n}-\mathbf{k})$ com a finalidade de reduzir o universo de busca e permitir que a determinação de novos códigos seja mais rápida. $O$ objetivo, agora, é encontrar as sub-matrizes $\mathbf{P}$, $\mathbf{M}_{0}$ e $\mathbf{N}_{0}$, de modo a otimizar a distância Euclidiana mínima do código. A sub-matriz $\mathbf{N}_{0}$ foi introduzida para permitir a obtenção de melhores códigos.

Para a extensão a uma modulação 8-PSK, propõe-se a utilização de códigos já determinados para a modulação 4-PSK acrescidos de submatrizes cíclicas, assim forma-se a matriz geradora 6.2. Neste caso, já conhecidas $\mathbf{P}, \mathbf{M}_{0}$ e $\mathbf{N}_{0}$, buscam-se apenas as sub-matrizes $\mathbf{M}_{1}$ e $\mathbf{N}_{1}$.

Nota-se que somente é necessário conhecer a primeira linha de cada submatriz $\mathbf{P}, \mathbf{M}_{\mathrm{i}}$ e $\mathbf{N}_{\mathrm{i}}$ para sua definição completa. A linha $(i+1)$ da sub-matriz é igual a linha $\mathrm{i}$ deslocada ciclicamente de um bit para a direita.

\section{CÓDIGOS ENCONTRADOS}

Utilizando-se busca computacional, foram encontrados novos códigos de bloco para modulação codificada. Esses códigos estão representados pelas sub-matrizes cíclicas binárias, formadoras de $\mathbf{G}^{\prime}$, nas Tabelas I e II, onde a primeira linha das sub-matrizes $\mathbf{P}, \mathbf{M}_{\mathrm{i}}$ e $\mathbf{N}_{\mathrm{i}}$ é dada por sua representação decimal. Por exemplo, $\mathbf{P}=3$ corresponde a linha (1100), com o bit mais significativo à direita.

A tabela $I$ apresenta códigos de taxa $\mathbf{R}=1 / 2$, para a modulação 4-PSK, e a Tabela II para a modulação 8-PSK.
Por exemplo, na Tabela I, o código com $\mathbf{n}=8, \mathrm{P}=3, \mathbf{M}_{0}$ $=4 \mathrm{e} \mathbf{N}_{0}=13$, fornece a seguinte matriz geradora:

$$
\mathbf{G}^{\prime}=\left|\begin{array}{llllllllllllllll}
1 & 0 & 0 & 0 & 0 & 0 & 0 & 0 & 1 & 1 & 0 & 0 & 0 & 0 & 1 & 0 \\
0 & 1 & 0 & 0 & 0 & 0 & 0 & 0 & 0 & 1 & 1 & 0 & 0 & 0 & 0 & 1 \\
0 & 0 & 1 & 0 & 0 & 0 & 0 & 0 & 0 & 0 & 1 & 1 & 1 & 0 & 0 & 0 \\
0 & 0 & 0 & 1 & 0 & 0 & 0 & 0 & 1 & 0 & 0 & 1 & 0 & 1 & 0 & 0 \\
0 & 0 & 0 & 0 & 1 & 0 & 0 & 0 & 1 & 0 & 1 & 1 & 1 & 1 & 0 & 0 \\
0 & 0 & 0 & 0 & 0 & 1 & 0 & 0 & 1 & 1 & 0 & 1 & 0 & 1 & 1 & 0 \\
0 & 0 & 0 & 0 & 0 & & 1 & 0 & 1 & 1 & 1 & 0 & 0 & 0 & 1 & 1 \\
0 & 0 & 0 & 0 & 0 & 0 & 0 & 1 & 0 & 1 & 1 & 1 & 1 & 0 & 0 & 1
\end{array}\right|
$$

Observa-se que os códigos da Tabela I, para 4-PSK, apresentam distâncias Euclidianas mínimas iguais às dos códigos pseudo-cíclicos encontrados por Baldini. Já os códigos da Tabela II, para 8-PSK, apresentam distâncias Euclidianas maiores que as dos códigos pseudo-cíclicos. As tabelas apresentam também a distância de Hamming do código binário de matriz G'.

\section{CONSIDERAÇÕES FINAIS}

Este trabalho apresentou um novo sistema para a obtençāo de códigos de bloco para a modulação codificada. Mostrou-se como representar o sistema por uma única matriz e, assim, uma nova forma de mapear um único código binário para o espaco de sinais, de modo a obter novos códigos. Uma vantagem do sistema apresentado é a utilização de um único codificador na transmissão e um único decodificador na recepção.

O processo de busca de códigos preocupa-se em maximizar a distância Euclidiana mínima do código. A busca de códigos consiste na alteração de apenas duas sub-matrizes cíclicas. Alguns códigos novos foram apresentados, e estes apresentam ganhos iguais ou maiores que aqueles que podem ser obtidos pelo método de Sayegh ou de Baldini.

Simulações realizadas em computador, com um código de $\mathbf{n}=8, \mathrm{~d}_{\mathrm{E}}^{2}=10$ e $\mathrm{g}_{\infty}=4 \mathrm{~dB}$, para modulação 4-PSK codificada (matriz 7.1), mostraram um ganho de $2 \mathrm{~dB}$ em relação a modulação 2-PSK não codificada a uma taxa de erro de bit de $10^{-3}$. Considerou-se o canal como sendo perturbado por um ruído aditivo, gaussiano e branco (AWGN). O processo de decodificação utilizado foi o do algoritmo de conjuntos de informação [8] com 32 matrizes de correção e decisão suave. Com relação à decodificação de máxima verossimilhança, há uma perda de $0,5 \mathrm{~dB}$ à mesma taxa de erro de bit $[6,7]$.

\section{REFERÊNCIAS}

[1] Ungerboeck, G.; "Channel Coding with Multilevel/Phase Signals", IEEE T. Inf. Theory, p. 55-67, janeiro, 1982.

[2] Imai, H. \& Hirakawa, S. "A New Multilevel Coding Method Using Error-Correcting Codes", IEEE Trans. on Inf. Theory, p. 371-377, maio, 1977. 
[3] Cusack, B. L., "Error Control for QAM Signalling", for Digital Communication", New York, 1981.

Electronics Letters, p. 62-63, janeiro, 1984.

[4] Sayegh, S.L.; "A Class of Optimum Block Codes in Signal Space", IEEE Trans. Comm., p. 1043-1045, outubro, 1986.

[5] Baldini Filho, R. "Códigos Pseudo-cíclicos Multiniveis", Anais do $7^{0}$ Simp. Bras. de Telecom., Florianópolis - SC, p. 402-405, setembro, 1989.

[6] Wille, E.C.G. \& Godoy Jr., W.; "Códigos Multiniveis para a Modulação Codificada", Anais do $9^{\circ}$ Simp. Bras. de Telecom., São Paulo - SP, p.341-345, 1991.

[7] Wille, E.C.G. \& Godoy Jr., W.; "Codificação, Busca e Decodificação de uma Classe de Códigos Binários no Espaco de Sinais", TELEMO'92, p. 623-628, Brasilia - DF, 1992.

[8] Clark, G. C. \& Cain, J. B.; "Error-Correction Coding TABELA I - CÓDIGOS PARA 4-PSK

\begin{tabular}{|c|c|c|c|c|c|}
\hline $\mathbf{n}$ & $\overrightarrow{\mathbf{P}}$ & $\mathbf{M}_{0}$ & $\mathbf{N}_{0}$ & $d^{\prime}$ & $\mathrm{d}_{\mathrm{E}}{ }^{2}$ \\
\hline 4 & 1 & $\begin{array}{l}2 \\
2 \\
3\end{array}$ & $\begin{array}{l}0 \\
3 \\
0\end{array}$ & $\begin{array}{l}2 \\
3 \\
2\end{array}$ & 8 \\
\hline 4 & 2 & $\begin{array}{l}1 \\
1 \\
3 \\
\end{array}$ & $\begin{array}{l}0 \\
3 \\
0 \\
\end{array}$ & $\begin{array}{l}2 \\
3 \\
2 \\
\end{array}$ & 8 \\
\hline 6 & 3 & $\begin{array}{l}4 \\
4 \\
4 \\
7 \\
7 \\
7 \\
\end{array}$ & $\begin{array}{l}1 \\
2 \\
7 \\
1 \\
2 \\
4\end{array}$ & 4 & 8 \\
\hline 6 & 5 & $\begin{array}{l}2 \\
2 \\
2 \\
7 \\
7 \\
7\end{array}$ & $\begin{array}{l}1 \\
4 \\
7 \\
1 \\
2 \\
4\end{array}$ & 4 & 8 \\
\hline 8 & 3 & $\begin{array}{c}4 \\
4 \\
4 \\
7 \\
8 \\
8 \\
8 \\
11 \\
13 \\
13 \\
14 \\
14\end{array}$ & $\begin{array}{c}6 \\
7 \\
13 \\
12 \\
9 \\
11 \\
14 \\
12 \\
1 \\
4 \\
2 \\
8\end{array}$ & 4 & 10 \\
\hline 10 & 3 & $\begin{array}{c}9 \\
9 \\
9 \\
10 \\
12 \\
13 \\
17 \\
18 \\
20 \\
20 \\
20 \\
21 \\
27 \\
30 \\
\end{array}$ & $\begin{array}{c}6 \\
15 \\
17 \\
6 \\
5 \\
10 \\
5 \\
3 \\
3 \\
12 \\
23 \\
18 \\
9 \\
20\end{array}$ & 6 & 12 \\
\hline
\end{tabular}

TABELA II - CÓDIGOS PARA 8-PSK

\begin{tabular}{|c|c|c|c|c|c|c|c|}
\hline $\mathbf{n}$ & $\mathbf{P}$ & $\mathbf{M}_{0}$ & $\mathbf{N}_{0}$ & $\mathbf{M}_{1}$ & $N_{1}$ & $\mathrm{~d}^{\prime}$ & $d_{E}{ }^{2}$ \\
\hline 4 & 1 & 2 & 3 & $\begin{array}{l}0 \\
3 \\
\end{array}$ & 1 & 3 & 3,17 \\
\hline 4 & 2 & 1 & 3 & $\begin{array}{l}0 \\
3\end{array}$ & 2 & 3 & 3,17 \\
\hline 4 & 2 & I & 0 & 1 & 3 & 3 & 4,58 \\
\hline 6 & 3 & 4 & 1 & 7 & 3 & 3 & 5,17 \\
\hline 6 & 3 & 4 & 2 & 7 & 3 & 3 & 5,17 \\
\hline 6 & 3 & 7 & 4 & $\begin{array}{l}5 \\
6\end{array}$ & $\begin{array}{l}6 \\
5\end{array}$ & 3 & 5,17 \\
\hline 8 & 3 & 4 & 13 & 8 & $\begin{array}{l}0 \\
1\end{array}$ & $\begin{array}{l}3 \\
5 \\
\end{array}$ & $\begin{array}{l}5,17 \\
4,68\end{array}$ \\
\hline 8 & 3 & 8 & 14 & 4 & $\begin{array}{l}0 \\
2 \\
\end{array}$ & $\begin{array}{l}3 \\
5\end{array}$ & $\begin{array}{l}5,17 \\
4,68 \\
\end{array}$ \\
\hline
\end{tabular}

Walter Godoy Júnior nasceu em Araçatuba, São Paulo, Brasil, em 1950. Ele recebeu o grau de M.Sc. no Instituto de Engenharia de Comunicações, St. Petersburg, Rússia e o grau de Doutor em Engenharia Elétrica na Universidade Estadual de Campinas (Unicamp), Brasil, em 1977 e 1990, respectivamente.

De 1979 a 1987 ele trabalhou na Telecomunicaçōes do Paraná (TELEPAR) em Curitiba, Brasil como Engenheiro de Pesquisa no Grupo de Radio-Relay Links.

Ele junto-se ao Departamento de Eletrônica, Centro Federal de Educação Tecnológica de Paraná (o CEFETPR), Curitiba, Brasil em 1979 como Professor Assistente, e desde 1991 ele tem o grau de Professor Sênior. Os seus principais campos de pesquisa são Códigos de Controle de Erro e suas aplicações, e Criptografia.

Prof. Godoy é um sócio de Sociedade Brasileira de Telecomunicações (SBT).

Emilio Carlos Gomes Wille nasceu em Lapa, Paraná, Brasil em 1965. Ele recebeu os títulos de Engenheiro Elétrico e Mestre em Eng. Elétrica do Centro Federal de Educação Tecnológica de Paraná (CEFET/PR), Curitiba, Brasil, em 1989 e 1991 respectivamente. Ele tem sido assistente de ensino e pesquisa no Departamento de Eletrônica de CEFET/PR, desde 1991 e participa do ensino de graduação e pós-graduação. Os seus interesses de pesquisa incluem teorias de codificação $e$ de modulação e modelagem/simulação de sistemas de comunicação. Ele está trabalhando agora em uma LAN que usa os circuitos de distribuição de energia elétrica como um canal de comunicação. 